\title{
Achados audiológicos em crianças com fenilcetonúria
}

\section{Audiologic findings in children with phenylketonuria}

\author{
Patrícia Cotta Mancini' ${ }^{1}$ Ana Lúcia Pimenta Starling², Letícia Macedo Penna ${ }^{3}$, Camila Alexandra Vilaça Ramos ${ }^{4}$, \\ Michelle Imaculada Otaviani Ferreira ${ }^{5}$, Maria Cecília Martinelli Iório $^{6}$
}

\begin{abstract}
RESUMO
Objetivo: Investigar a existência de alterações na audição de crianças com fenilcetonúria diagnosticadas e tratadas precocemente e comparar os resultados com os encontrados nas avaliações auditivas de crianças normais de mesma idade. Métodos: Foram realizadas imitanciometria e audiometria tonal e vocal em 63 crianças, sendo 30 no grupo controle, com média de idade de 8,1 anos, e 33 com fenilcetonúria no grupo de estudo, com média de idade de 7,7 anos. O grupo de estudo foi subdividido em 15 crianças com controle adequado da dieta e 18 crianças com controle inadequado da dieta, com médias de idade 8,1 e 7,2, respectivamente. A análise estatística utilizou o Teste t ou ANOVA. Resultados: A audiometria revelou 83,3\% de crianças com audição normal no grupo controle e $16,7 \%$ de perdas auditivas condutivas uni ou bilaterais. No grupo com fenilcetonúria, 66,7\% das crianças apresentaram audição normal e 33,3\% com perdas auditivas condutivas. Na imitanciometria, observou-se curvas normais em $91,7 \%$ das crianças do grupo controle e em $72,7 \%$ das crianças do grupo com fenilcetonúria. Houve diferença na comparação entre grupos para limiares aéreos, reflexos estapedianos, limiares de recepção da fala e índice de reconhecimento de fala. Não foi observada diferença entre os resultados das avaliações auditivas de crianças fenilcetonúricas com dieta adequada e inadequada. Conclusão: As crianças com fenilcetonúria diagnosticadas e tratadas precocemente apresentaram piores limiares de audibilidade por via aérea, limiares de recepção de fala e índice de reconhecimento de fala evidenciados à audiometria tonal e vocal, quando comparadas com crianças normais.
\end{abstract}

Descritores: Audição; Fenilcetonúria; Transtornos da audição; Testes de impedância acústica; Perda auditiva; Audiometria

\section{INTRODUÇÃO}

A fenilcetonúria (PKU) é uma doença genética de caráter autossômico recessivo, causada pela deficiência de atividade da fenilalanina hidroxilase hepática, enzima que metaboliza a fenilalanina em outro aminoácido, a tirosina ${ }^{(1,2)}$. Esse erro metabólico ocasiona acúmulo de fenilalanina no sangue e outros

Trabalho realizado no Serviço de Audiologia do Hospital das Clínicas da Universidade Federal de Minas Gerais - UFMG - Belo Horizonte (MG), Brasil. (1) Pós-graduanda (Doutorado) em Ciências dos Distúrbios da Comunicação Humana: Campo Fonoaudiológico pela Universidade Federal de São Paulo - UNIFESP - São Paulo (SP), Brasil; Professora Assistente do Curso de Fonoaudiologia da Universidade Federal de Minas Gerais - UFMG - Belo Horizonte (MG), Brasil.

(2) Doutora, Professora Adjunto do Departamento de Pediatria da Faculdade de Medicina da Universidade Federal de Minas Gerais - UFMG - Belo Horizonte (MG), Brasil.

(3) Professora Substituta do Curso de Fonoaudiologia da Universidade Federal de Minas Gerais - UFMG - Belo Horizonte (MG), Brasil.

(4) Acadêmica do Curso de Fonoaudiologia da Universidade Federal de Minas Gerais - UFMG - Belo Horizonte (MG), Brasil.

(5) Acadêmica do Curso de Fonoaudiologia da Universidade Federal de Minas Gerais - UFMG - Belo Horizonte (MG), Brasil.

(6) Doutora, Professora Associada do Curso de Fonoaudiologia da Universidade Federal de São Paulo - UNIFESP - São Paulo (SP), Brasil.

Endereço para correspondência: Patrícia Cotta Mancini. R. Himalaia, 23, Vale do Sol, Nova Lima (MG), Brasil, CEP: 34000-000. E-mail: patmancini@gmail.com

Recebido em: 16/7/2009; Aceito em: 7/11/2009 líquidos corporais, incluindo o líquor (que determina alterações bioquímicas no sistema nervoso central - SNC), prejudicando o processo de mielinização e causando deficiência na síntese de proteínas e de neurotransmissores ${ }^{(3)}$. Quando não tratada em tempo adequado, a PKU resulta em alterações importantes no SNC, manifestadas em distúrbios da locomoção e da fala, hiperatividade, tremor, microcefalia, alterações no crescimento e, principalmente, retardo mental de grau e intensidade variados, sempre irreversível ${ }^{(2,4-6)}$.

O tratamento precoce ideal deve ser realizado preferencialmente até os primeiros 21 dias de vida, evitando as manifestações clínicas e bioquímicas da doença ${ }^{(2,7,8)}$. Esse tratamento consiste em uma dieta restrita em fenilalanina a fim de manter as concentrações sanguíneas do aminoácido dentro dos limites adequados para a faixa etária. As concentrações de fenilalanina no sangue consideradas de referência para fenilcetonúricos são: de 120 a $360 \mu \mathrm{mol} / \mathrm{L}$ para lactentes e pré-escolares, até $480 \mu \mathrm{mol} / \mathrm{L}$ para escolares e até $700 \mu \mathrm{mol} / \mathrm{L}$ para adolescentes ${ }^{(7-9)}$. Normalmente o aleitamento materno é eliminado ou reduzido e na dieta é utilizada uma mistura de aminoácidos, administrada por meio de mamadeira, isenta ou com pequena quantidade de fenilalanina para garantir o aporte protéico adequado necessário para o crescimento e desenvolvimento da criança, uma vez que a ingestão de proteínas naturais é controlada ${ }^{(3,6)}$. O tratamento deve ser mantido por toda a vida, e qualquer descuido em relação à ingestão de fenilalanina pode 
ocasionar déficits na função neuropsicológica e no processo de mielinização do sistema nervoso, como demonstrado por imagens de ressonância magnética de fenilcetonúricos ${ }^{(3)}$.

O diagnóstico precoce da PKU é possível pelo teste de triagem neonatal ("Teste do Pezinho"), no qual se dosa a fenilalanina no sangue no quinto dia de vida ${ }^{(8)}$. Quando a dosagem encontra-se maior ou igual a $240 \mu \mathrm{mol} / \mathrm{L}$ a criança é encaminhada para diagnóstico diferencial e tratamento essencialmente dietético, se necessário ${ }^{(6,8)}$.

A PKU ocorre em todos os grupos étnicos com incidência diversificada, variando entre os recém-nascidos de 1:2.600 até 1:26.000, sendo a média de 1:10.000 ${ }^{(3-5)}$. Em Minas Gerais, a incidência é de 1:21.000 recém-nascidos vivos ${ }^{(10)}$.

Quando a dieta é iniciada nas primeiras semanas de vida e mantida continuamente, os indivíduos com PKU desenvolvemse normalmente. Entretanto, foram observados déficits nas funções executivas mesmo em crianças cujo tratamento é iniciado precocemente e mantido de forma ininterrupta ${ }^{(1)}$. As funções executivas envolvem as habilidades de planejamento, memória operacional, inibição e auto-regulação do comportamento, estando associadas ao funcionamento das porções dorso-laterais do lobo pré-frontal ${ }^{(11)}$. Outros estudos apontaram déficits na interação inter-hemisférica cerebral até mesmo em crianças diagnosticadas e tratadas precocemente ${ }^{(12)}$, além de déficits cognitivos em várias áreas (atenção, memória, aprendizagem e funções executivas $)^{(13)}$. Portanto, ainda são questionados os níveis plasmáticos ideais de fenilalanina a fim de evitar os efeitos neurológicos em longo prazo, observados em estudos mais recentes $^{(3)}$.

A PKU é a mais comum das doenças envolvendo erros congênitos no metabolismo de aminoácidos e foi inicialmente descrita em $1934^{(14)}$.

Em 1962, foram relatados dois casos nos quais os pais de crianças com PKU ainda não diagnosticadas suspeitavam que seus filhos fossem surdos ou que apresentassem alguma dificuldade de audição. Apesar dessa suspeita, não foi detectada nenhuma perda auditiva pela audiometria condicionada realizada na época ${ }^{(15)}$.

Mais recentemente, outro estudo investigou a relação entre desempenho escolar e funções cognitivas, sendo avaliada a memória auditiva e habilidades de aprendizado auditivo-verbal em 26 crianças com PKU tratadas precocemente e comparadas com 21 crianças sem PKU ${ }^{(16)}$. Nesse estudo, não foi encontrada diferença significativa entre alteração na memória auditiva e problemas escolares quando os pacientes com PKU tratados precocemente foram comparados com o grupo controle, já que apenas um paciente do grupo de estudo apresentou alteração na memória auditiva.

A proposta deste estudo surgiu da necessidade de se avaliar a audição de crianças com PKU, uma vez que a equipe multidisciplinar relatava queixas auditivas frequentes nesses pacientes, feitas pelos pais ou pelos responsáveis. A revisão da literatura aponta para a escassez de trabalhos sobre o funcionamento do sistema auditivo em crianças com PKU. O objetivo deste estudo é investigar a existência de alterações na audição de crianças com fenilcetonúria diagnosticadas e tratadas precocemente e comparar os resultados com os encontrados nas avaliações auditivas de crianças normais de mesma idade.

\section{MÉTODOS}

O estudo transversal foi aprovado pelo Comitê de Ética em Pesquisa da Universidade Federal de Minas Gerais (UFMG), sob parecer $n^{\circ} 0895 / 07$. Os pais ou os responsáveis pelas crianças foram contatados e informados sobre o caráter voluntário da pesquisa, sendo solicitados a permitir a participação do seu filho no estudo. Foram fornecidas as informações sobre os procedimentos a serem realizados e sobre a ausência de qualquer risco à saúde durante os procedimentos. Os responsáveis pelas crianças consentiram com a realização da pesquisa e a divulgação de seus resultados, assinando o Termo de Consentimento Livre e Esclarecido, conforme a resolução 196/96 do Ministério da Saúde. Os exames foram então agendados e os dados foram coletados entre agosto de 2007 e maio de $2008 \mathrm{em}$ um Serviço de Audiologia, onde um fonoaudiólogo realizou os exames, sem saber a qual grupo pertencia cada criança.

Todas as crianças foram submetidas à inspeção do meato acústico externo seguido de imitanciometria (classificação das curvas timpanométricas ${ }^{(17)}$ e pesquisa dos reflexos acústicos) por meio do imitanciômetro Interacoustics ${ }^{\circledR}$ AZ7. As curvas do tipo A foram consideradas normais e as demais configurações foram consideradas alteradas. Os reflexos estapedianos foram obtidos contralateralmente em ambas as orelhas nas frequências de $500 \mathrm{~Hz}, 1,2$ e $4 \mathrm{kHz}$. Para fins de análise, foi considerado o valor de $125 \mathrm{~dB}$ quando o reflexo estava ausente, uma vez que a saída máxima do equipamento para a mensuração dos reflexos contralaterais é $120 \mathrm{~dB}$. Em seguida foi realizada a audiometria tonal e vocal, em cabine tratada acusticamente, utilizando o audiômetro Interacoustics modelo AD 229b, fones supra-aurais TDH 39 e vibrador B71 para determinação dos limiares tonais aéreos (LA), ósseos (LO) e logoaudiométricos, bem como do índice de reconhecimento de fala (IRF) e limiar de reconhecimento de fala (LRF) de cada orelha. A classificação das audiometrias considerou a média dos limiares de audibilidade nas frequências de $500 \mathrm{~Hz}, 1,2$ e $4 \mathrm{kHz}^{(18)}$, sendo considerado o limite de normalidade de 15 dB para essa média.

Participaram deste estudo 63 crianças e adolescentes, com idade variando entre quatro e 15 anos, de ambos os sexos, sem determinação de cor ou raça. O grupo controle foi constituído por 30 crianças e adolescentes sem PKU ou qualquer outra alteração sensorial, neuro-motora ou psico-cognitiva evidente, saudáveis, recrutadas entre os pacientes do Serviço de Pediatria Geral Hospital das Clínicas da UFMG, que estavam aguardando consulta de rotina. Esse grupo foi composto por 14 crianças do gênero masculino e 16 do gênero feminino, com média de idade de 8,1 anos. O grupo de estudo (com PKU) contou com 33 crianças e adolescentes, todas diagnosticadas e tratadas precocemente, sendo 14 do gênero feminino e 19 do gênero masculino, com média de idade de 7,67 anos. Esse grupo foi dividido em dois subgrupos, a saber: Grupo A, composto por 15 crianças com PKU com média de idade de 8,13 anos, que foram consideradas como tendo controle adequado da dieta; e Grupo I, composto por 18 crianças com PKU com média de idade de 7,22 anos, mas que foram consideradas como tendo controle inadequado da dieta. As crianças tinham controle dietético adequado quando $70 \%$ ou mais de todas as dosagens 
sanguíneas de fenilalanina, realizadas após o diagnóstico da PKU, estavam dentro dos limites preconizados para fenilcetonúricos, naquela faixa etária. Os pacientes do grupo de estudo estão em tratamento no Ambulatório de Fenilcetonúria do Serviço Especial de Genética do HC-UFMG, parceiro do Núcleo de Ações e Pesquisa em Apoio Diagnóstico (NUPAD) da Faculdade de Medicina da UFMG, serviço de referência de Triagem Neonatal do Estado de Minas Gerais.

Os resultados foram analisados e comparados a fim de investigar possíveis alterações encontradas na audição das crianças de todos os grupos. O banco de dados foi discutido entre os pesquisadores e estruturado a partir do programa Excel, sendo as análises estatísticas realizadas com o programa estatístico SPSS (Statistical Package for the Social Sciences) versão 13.0.

Estatística descritiva incluindo índices de medida de tendência central (média) e de dispersão (desvio padrão), bem como frequência, foi usada para caracterizar as crianças de todos os grupos em relação a variáveis demográficas, sendo também utilizados para descrever as respostas obtidas em cada grupo.

Análises inferenciais das variáveis dependentes quantitativas foram precedidas de teste de normalidade das distribuições em cada grupo. No caso de apresentarem distribuição normal, foram usados o Teste t para grupos independentes e a ANOVA. No caso das distribuições serem diferentes de uma distribuição gaussiana, os testes não-paramétricos de Wilcoxon e MannWhitney U foram usados para comparação entre grupos. Para as variáveis dependentes qualitativas, a comparação entre grupos foi realizada com o teste Qui-quadrado. Em todas as análises foi considerado nível de significância $\alpha=0,05$.

Nesse estudo, a análise dos LA, LO e reflexos estapedianos revelou distribuição normal, sendo utilizado o teste-t para comparação entre grupos controle e PKU, ou ANOVA quando a comparação foi realizada entre os grupos controle, PKU com dieta adequada e PKU com dieta inadequada. O LRF e IRF não apresentaram distribuição normal, sendo, portanto, utilizado os testes não-paramétricos de Wilcoxon e Mann-Whitney U para a comparação entre grupos. O Qui-quadrado foi utilizado para análise das curvas timpanométricas.

\section{RESULTADOS}

A classificação das audiometrias quanto ao tipo de perda auditiva revelou que, no grupo controle, $83,3 \%$ das crianças apresentaram exames normais bilateralmente, enquanto que no grupo com PKU 66,7\% das crianças tiveram exames normais. As perdas auditivas foram do tipo condutivo, discretas ou leves, uni ou bilaterais em 16,7\% do grupo controle e em $33,3 \%$ das crianças com PKU. As perdas auditivas mistas ou neurossensoriais não ocorreram em nenhum grupo. Apesar das crianças com PKU apresentarem mais audiometrias alteradas, o teste do Qui-quadrado não revelou diferença na comparação entre grupos $(\mathrm{p}=0,113)$.

A imitanciometria foi analisada em número de orelhas, sendo as curvas normais (tipo A) observadas em 91,7\% das orelhas do grupo controle e em 72,7\% do grupo com PKU. No grupo estudo com dieta adequada foram encontradas $76,7 \%$ de curvas tipo A, $3,3 \%$ de curvas tipo B e $20 \%$ de curvas do tipo C, enquanto que no grupo com dieta inadequada foram observadas $69,4 \%$ de curvas tipo A, 2,8\% de curvas tipo B e 27,8\% de curvas tipo C. No grupo controle verificou-se $8,3 \%$ de curvas do tipo $\mathrm{C}$ e nenhum caso de curva do tipo B. A análise estatística revelou que não há associação entre os dois grupos em relação ao tipo de curva timpanométrica $(\mathrm{p}=0,181)$ (Tabela 1$)$.

Em seguida, os limiares aéreos foram analisados por frequência em cada orelha (Tabela 2 e Figura 1). Constatou-se a mesma tendência para todos os grupos de possuírem limiares mais elevados nas frequências de $250,500 \mathrm{~Hz}$ e $8 \mathrm{kHz}$, independente da orelha. A análise estatística não revelou diferença na comparação entre as orelhas direita e esquerda em nenhuma das frequências testadas na audiometria, em nenhum grupo $(\mathrm{p}=0,436)$.

Prosseguiu-se o estudo considerando os limiares de audibilidade médios (média de $500 \mathrm{~Hz}, 1,2$ e $4 \mathrm{kHz}$ ), não sendo encontrada diferença na comparação entre as orelhas direita e esquerda para cada grupo. Também não foi observada diferença na comparação entre as orelhas direita e esquerda para LO, IRF e LRF (Tabela 3).

Dessa forma, procedeu-se então à comparação dos valores médios dessas medidas entre os três grupos. Como não foi

Tabela 1. Classificação das audiometrias e curvas timpanométricas, bem como dados descritivos da idade e sexo para o grupos controle e estudo

\begin{tabular}{|c|c|c|c|c|}
\hline \multirow[t]{2}{*}{ Parâmetros } & & \multirow[t]{2}{*}{ Grupo controle $(\mathrm{N}=30)$} & \multicolumn{2}{|c|}{ Grupo estudo } \\
\hline & & & Grupo A (N=15) & Grupo I $(\mathrm{N}=18)$ \\
\hline Média de idade (DP) & & $8,10(3,04)$ & $8,13(87)$ & $7,22(2,16)$ \\
\hline \multirow[t]{2}{*}{ Gênero - N (\%) } & Masculino & $14(46,7)$ & $10(66,7)$ & $9(50)$ \\
\hline & Feminino & $16(53,3)$ & $5(33,3)$ & $9(50)$ \\
\hline \multirow[t]{3}{*}{ Audiometria - N (\%) } & Normais & $25(83,3)$ & $10(66,7)$ & $12(66,7)$ \\
\hline & PA cond. unilaterais & $3(10)$ & $4(26,7)$ & $2(11,1)$ \\
\hline & PA cond. bilaterais & $2(6,7)$ & $1(6,6)$ & $4(22,2)$ \\
\hline \multirow[t]{3}{*}{ Imitanciometria orelhas (\%) } & Tipo A & $55(91,7)$ & $23(76,7)$ & $25(69,4)$ \\
\hline & Tipo B & $0(0)$ & $1(3,3)$ & $1(2,8)$ \\
\hline & Tipo C & $5(8,3)$ & $6(20)$ & $10(27,8)$ \\
\hline
\end{tabular}

Legenda: Grupo A = crianças com fenilcetonúria e dieta adequada; Grupo I = crianças com fenilcetonúria e dieta inadequada; N = número de participantes; PA = perdas auditivas; $\mathrm{DP}$ = desvio-padrão; cond. = condutiva 
Tabela 2. Limiares de audibilidade médios (dBNA) por frequência nas orelhas direita e esquerda, nos grupos controle e estudo

\begin{tabular}{|c|c|c|c|c|c|c|c|}
\hline \multirow[t]{3}{*}{ Orelha } & \multirow[t]{3}{*}{ Frequência } & \multirow{2}{*}{\multicolumn{2}{|c|}{$\begin{array}{l}\text { Grupo controle } \\
\qquad(\mathrm{N}=30)\end{array}$}} & \multicolumn{4}{|c|}{ Grupo estudo } \\
\hline & & & & \multicolumn{2}{|c|}{ Grupo A (N=15) } & \multicolumn{2}{|c|}{ Grupo I $(\mathrm{N}=18)$} \\
\hline & & Média & $\mathrm{DP}$ & Média & DP & Média & $\mathrm{DP}$ \\
\hline \multirow[t]{6}{*}{ OD } & $250 \mathrm{~Hz}$ & 15,33 & 6,15 & 17,33 & 8,63 & 18,61 & 8,19 \\
\hline & $500 \mathrm{~Hz}$ & 14,67 & 5,86 & 18,33 & 7,72 & 18,06 & 8,60 \\
\hline & $1 \mathrm{kHz}$ & 10,83 & 5,43 & 11,00 & 7,12 & 12,78 & 7,32 \\
\hline & $2 \mathrm{kHz}$ & 8,33 & 6,48 & 10,00 & 6,27 & 8,33 & 7,07 \\
\hline & $4 \mathrm{kHz}$ & 9,83 & 5,80 & 8,33 & 5,56 & 10,00 & 6,42 \\
\hline & $8 \mathrm{kHz}$ & 14,33 & 6,40 & 14,00 & 7,61 & 18,06 & 4,25 \\
\hline \multirow[t]{6}{*}{ OE } & $250 \mathrm{~Hz}$ & 14,33 & 6,40 & 17,00 & 7,75 & 17,22 & 10,46 \\
\hline & $500 \mathrm{~Hz}$ & 11,83 & 6,76 & 17,00 & 9,22 & 19,72 & 11,18 \\
\hline & $1 \mathrm{kHz}$ & 8,83 & 6,11 & 10,33 & 7,43 & 11,94 & 6,67 \\
\hline & $2 \mathrm{kHz}$ & 8,17 & 8,25 & 9,67 & 7,19 & 9,72 & 5,28 \\
\hline & $4 \mathrm{kHz}$ & 8,67 & 9,19 & 9,67 & 7,90 & 11,39 & 7,82 \\
\hline & $8 \mathrm{kHz}$ & 10,67 & 6,66 & 15,33 & 8,76 & 15,56 & 5,66 \\
\hline
\end{tabular}

Legenda: $\mathrm{OD}$ = orelha direita; $\mathrm{OE}$ = orelha esquerda; Grupo $\mathrm{A}=$ crianças com fenilcetonúria e dieta adequada; Grupo I = crianças com fenilcetonúria e dieta inadequada; $\mathrm{N}$ = número de participantes; $\mathrm{DP}$ = desvio-padrão
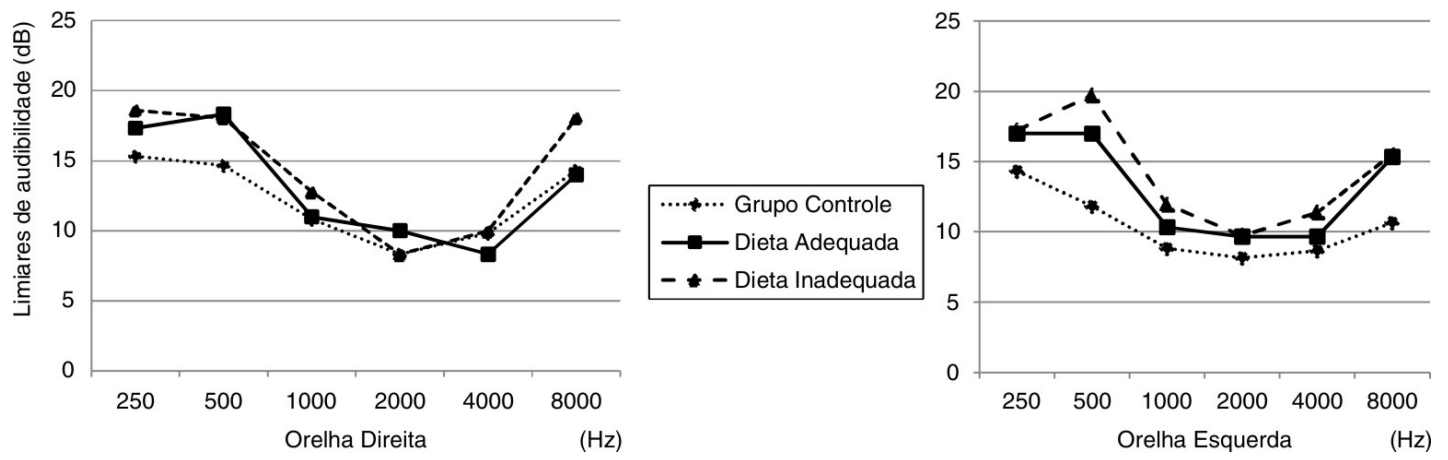

Figura 1. Limiares de audibilidade médios (dBNA) por frequência nas orelhas direita e esquerda, nos grupos controle e estudo

observada diferença na comparação entre os grupos de PKU com dieta adequada e inadequada, prosseguiu-se a análise considerando apenas dois grupos (controle e PKU). Nessa análise, observou-se diferença para LA, IRF e LRF, não sendo encontrada diferença apenas para LO (Tabela 4).

A seguir, os limiares dos reflexos estapedianos foram analisados, sendo considerado $125 \mathrm{~dB}$ quando o reflexo estava ausente, uma vez que a saída máxima do equipamento para a mensuração dos reflexos contralaterais é $120 \mathrm{~dB}$. A comparação entre as orelhas não revelou diferença (Tabela 5).

Como não foi observada diferença entre as orelhas, prosseguiu-se a análise sem considerar essa variável, sendo encontrada diferença significativa na comparação das médias dos reflexos estapedianos entre grupos $(\mathrm{p}=0,004)$. A análise post-hoc revelou que há diferença entre crianças do grupo controle e crianças do grupo PKU com dieta inadequada, sendo que essas últimas apresentam limiares de reflexos estapedianos mais elevados $(\mathrm{p}=0,003)$.

\section{DISCUSSÃO}

No presente estudo observou-se que há maior ocorrência de perdas condutivas em crianças com PKU, sendo esse achado mais evidente para perda condutiva bilateral no grupo com dieta inadequada. As perdas auditivas condutivas estiveram associadas às curvas timpanométricas alteradas. Sabe-se que crianças amamentadas por pelo menos três meses têm menor risco de otites ${ }^{(19)}$ e cabe destacar que as crianças do grupo PKU que participaram desse estudo foram privadas do aleitamento materno, devido ao protocolo que o serviço de referência para tratamento da PKU seguia à época do nascimento dessas crianças. A mamadeira foi introduzida tão logo a criança recebeu o diagnóstico, sendo mantida por tempo prolongado para facilitar a ingestão da fórmula de aminoácidos. Sabemos que o uso de mamadeira predispõe a criança à ocorrência de otites devido à posição na qual ela é oferecida aos bebês. Por outro lado, não foram coletados dados referentes ao tempo de aleitamento materno nas crianças do grupo controle, o que torna difícil estabelecer uma justificativa para a diferença encontrada nas curvas timpanométricas dos dois grupos. Enfim, as avaliações auditivas de crianças com PKU devem ser realizadas com atenção a fim de se estabelecer um correto diagnóstico audiológico, fazendo os encaminhamentos ao pediatra ou otorrinolaringologista quando necessário.

As crianças com PKU apresentaram piores LA e LRF, e acreditamos que tais achados devem estar relacionados à maior 
Tabela 3. Resultados do teste-t pareado para comparação dos limiares aéreos, ósseos, LRF e IRF médios obtidos por orelha, nos grupos controle e estudo

\begin{tabular}{|c|c|c|c|c|c|c|}
\hline Grupo & Variáveis & Média (DP) & Mediana & Mín & Máx & Valor de $p$ \\
\hline \multirow[t]{8}{*}{ Grupo controle $(\mathrm{N}=30)$} & LA OD (dBNA) & $10,92(4,59)$ & 11,88 & $-1,25$ & 20,00 & 0,216 \\
\hline & LA OE (dBNA) & $9,37(6,49)$ & 8,13 & $-1,25$ & 32,50 & \\
\hline & LO OD (dBNA) & $4,11(3,71)$ & 5,00 & $-5,00$ & 11,66 & 0,915 \\
\hline & LO OE (dBNA) & $4,11(4,10)$ & 5,00 & $-5,00$ & 11,66 & \\
\hline & LRF OD (dBNA) & $15,33(5,57)$ & 15,00 & 5,00 & 25,00 & 0,062 \\
\hline & LRF OE (dBNA) & $13,67(5,71)$ & 10,00 & 5,00 & 25,00 & \\
\hline & IRF OD (\%) & $97,07(3,63)$ & 100,00 & 88,00 & 100,00 & 0,677 \\
\hline & IRF OE (\%) & $97,33(3,21)$ & 98,00 & 88,00 & 100,00 & \\
\hline \multirow[t]{8}{*}{ Grupo A (N=15) } & LA OD (dBNA) & $11,92(5,40)$ & 11,25 & 3,75 & 25,00 & 0,815 \\
\hline & LA OE (dBNA) & $12,44(6,98)$ & 11,25 & 3,33 & 31,66 & \\
\hline & LO OD (dBNA) & $4,00(3,01)$ & 3,33 & $-1,66$ & 10,00 & 0,189 \\
\hline & LO OE (dBNA) & $3,66(2,97)$ & 3,33 & $-1,66$ & 10,00 & \\
\hline & LRF OD (dBNA) & $18,33(5,88)$ & 20,00 & 10,00 & 35,00 & 0,265 \\
\hline & LRF OE (dBNA) & $18,33(6,17)$ & 20,00 & 10,00 & 35,00 & \\
\hline & IRF OD (\%) & $95,20(4,06)$ & 96,00 & 88,00 & 100,00 & 0,991 \\
\hline & IRF OE (\%) & $93,87(3,96)$ & 92,00 & 88,00 & 100,00 & \\
\hline \multirow[t]{8}{*}{ Grupo I $(\mathrm{N}=18)$} & LA OD (dBNA) & $12,29(6,13)$ & 11,25 & 2,50 & 25,00 & 0,319 \\
\hline & LA OE (dBNA) & $13,19(6,26)$ & 13,13 & 3,75 & 27,50 & \\
\hline & LO OD (dBNA) & $3,98(4,92)$ & 3,33 & $-6,66$ & 13,33 & 0,997 \\
\hline & LO OE (dBNA) & $3,98(4,99)$ & 3,33 & $-6,66$ & 13,33 & \\
\hline & LRF OD (dBNA) & $18,61(8,01)$ & 15,00 & 10,00 & 35,00 & 0,439 \\
\hline & LRF OE (dBNA) & $20,00(9,55)$ & 17,50 & 5,00 & 40,00 & \\
\hline & IRF OD (\%) & $96,00(4,34)$ & 96,00 & 88,00 & 100,00 & 0,361 \\
\hline & IRF OE (\%) & $95,11(4,24)$ & 96,00 & 88,00 & 100,00 & \\
\hline
\end{tabular}

Legenda: Grupo A = crianças com fenilcetonúria e dieta adequada; Grupo I = crianças com fenilcetonúria e dieta inadequada; $\mathrm{N}$ = número de participantes; LA OD = média dos limiares aéreos da orelha direita; $L A$ OE = média dos limiares aéreos da orelha esquerda; LO OD = média dos limiares ósseos da orelha direita; LO OE = média dos limiares ósseos da orelha esquerda; LRF OD = limiares de recepção da fala da orelha direita; LRF OE = limiares de recepção da fala da orelha esquerda; IRF OD = índice de reconhecimento de fala da orelha direita; IRF OE = índice de reconhecimento de fala da orelha esquerda; Mín = valor mínimo observado; Máx = valor máximo observado

Tabela 4. Resultados do teste-t para comparação dos LA, LO, IRF e LRF entre os grupos controle e estudo ( $\mathrm{A}$ e I)

\begin{tabular}{lccc}
\hline Variável & $\begin{array}{c}\text { Grupo controle } \\
\text { Média (DP) }\end{array}$ & $\begin{array}{c}\text { Grupo estudo } \\
\text { Média (DP) }\end{array}$ & Valor de p \\
\hline LA (dBNA) & $10,15( \pm 4,53)$ & $12,31( \pm 5,68)$ & $0,035^{*}$ \\
LO (dBNA) & $4,11( \pm 3,85)$ & $3,91( \pm 4,10)$ & 0,845 \\
IRF (\%) & $97,2( \pm 2,95)$ & $95,03( \pm 3,57)$ & $0,011^{*}$ \\
LRF (dBNA) & $14,83( \pm 5,61)$ & $18,86( \pm 6,93)$ & $0,014^{*}$ \\
\hline
\end{tabular}

*Valores estatisticamente significantes $(p \leq 0,05)$ - Teste-t

Legenda: $L A$ = limiares de audibilidade por via aérea; $L O=$ limiares de audibilidade por via óssea; IRF = índice de reconhecimento de fala; LRF = limiar de recepção da fala

ocorrência de perdas condutivas observadas no grupo com PKU. Os limiares de audibilidade por via óssea não estiveram alterados, caracterizando as perdas auditivas condutivas encontradas.

Em relação aos reflexos estapedianos, as crianças com PKU e controle inadequado da dieta apresentaram limiares mais elevados quando comparadas às crianças do grupo controle. Alguns autores têm observado que indivíduos com distúrbio do processamento auditivo podem apresentar alterações nos reflexos estapedianos evidenciadas como ausência ou aumento dos limiares dos reflexos acústicos ${ }^{(20,21)}$, como ocorrido nas crianças com PKU. Desse modo, torna-se necessário avaliar o processamento auditivo dessas crianças a fim de diagnosticar e intervir o mais precocemente possível, uma vez que o adequado processamento da informação sensorial auditiva é imprescindível para a boa aquisição e desenvolvimento da linguagem.

A diferença observada para o IRF também deve ser estudada, uma vez que em perdas condutivas é esperado que o IRF esteja dentro dos padrões de normalidade, ou seja, acima de $88 \%$. O pior desempenho das crianças com PKU em relação ao IRF pode também indicar déficits no processamento da informação auditiva. Devido ao fato da PKU ser uma doença que causa deficiência na produção de neurotransmissores, torna-se necessária uma investigação das porções auditivas mais centrais a fim de descartar a possibilidade de lesão na via auditiva desses pacientes.

Considerando que a PKU é um erro congênito do metabolismo que acarreta alterações na mielinização, estudos dos potenciais evocados auditivos e do processamento auditivo devem ser conduzidos nessas crianças a fim de avaliar possíveis alterações subclínicas na via auditiva periférica e central, uma vez que os métodos de avaliação eletrofisiológica e comportamental podem detectar as alterações funcionais no sistema auditivo, não evidenciadas na audiometria convencional. Uma vez que o diagnóstico da PKU acontece precocemente, torna-se necessário investigar e conhecer os possíveis comprometimentos na audição dessas crianças. Identificar essas alterações em um estágio precoce possibilitará o desenvolvimento de novas abordagens terapêuticas e recursos auxiliares que minimizarão 
Tabela 5. Resultados do teste-t pareado para comparação dos reflexos estapedianos contralaterais médios obtidos por orelha, nos grupos controle e estudo

\begin{tabular}{|c|c|c|c|c|c|c|c|}
\hline \multirow[t]{2}{*}{ Frequência } & \multirow[t]{2}{*}{ Resultado } & \multicolumn{2}{|c|}{ Grupo controle } & \multicolumn{2}{|c|}{ Grupo A } & \multicolumn{2}{|c|}{ Grupo I } \\
\hline & & OD & OE & OD & $\mathrm{OE}$ & OD & $\mathrm{OE}$ \\
\hline \multirow[t]{4}{*}{$500 \mathrm{~Hz}$} & Média (DP) & $100,67( \pm 11,58)$ & $103( \pm 10,47)$ & $106,67( \pm 8,59)$ & $104,67( \pm 9,90)$ & $110( \pm 15,15)$ & $109,17( \pm 13,64)$ \\
\hline & Mín & 80 & 90 & 95 & 95 & 85 & 85 \\
\hline & Máx & 125 & 125 & 125 & 125 & 125 & 125 \\
\hline & Valor de $p$ & \multicolumn{2}{|c|}{0,08} & \multicolumn{2}{|c|}{0,384} & \multicolumn{2}{|c|}{0,579} \\
\hline \multirow[t]{4}{*}{$1 \mathrm{kHz}$} & Média (DP) & $99,83( \pm 11,02)$ & $101,33( \pm 12,17)$ & $103( \pm 8,62)$ & $103( \pm 8,41)$ & $108,89( \pm 1301)$ & $106,67( \pm 12,48)$ \\
\hline & Mín & 85 & 85 & 90 & 85 & 85 & 85 \\
\hline & Máx & 125 & 125 & 125 & 125 & 125 & 125 \\
\hline & Valor de $p$ & \multicolumn{2}{|c|}{0,370} & \multicolumn{2}{|c|}{$>0,999$} & \multicolumn{2}{|c|}{0,149} \\
\hline \multirow[t]{4}{*}{$2 \mathrm{kHz}$} & Média (DP) & $99( \pm 11,01)$ & $100,50( \pm 12,06)$ & $100,33( \pm 9,54)$ & $10033( \pm 8,96)$ & $111,39( \pm 12,34)$ & $108,61( \pm 11,73)$ \\
\hline & Mín & 85 & 80 & 85 & 85 & 95 & 95 \\
\hline & Máx & 125 & 125 & 125 & 125 & 125 & 125 \\
\hline & Valor de $p$ & \multicolumn{2}{|c|}{0,393} & \multicolumn{2}{|c|}{$>0,999$} & \multicolumn{2}{|c|}{0,299} \\
\hline \multirow[t]{4}{*}{$4 \mathrm{kHz}$} & Média (DP) & $99,17( \pm 12,53)$ & $103( \pm 14,06)$ & $106( \pm 12,13)$ & $103( \pm 10,66)$ & $112,78( \pm 1191)$ & $111,67( \pm 14,35)$ \\
\hline & Mín & 75 & 75 & 85 & 90 & 95 & 85 \\
\hline & Máx & 125 & 125 & 125 & 125 & 125 & 125 \\
\hline & Valor de $p$ & \multicolumn{2}{|c|}{0,475} & \multicolumn{2}{|c|}{0,094} & \multicolumn{2}{|c|}{0,123} \\
\hline
\end{tabular}

*Valores estatisticamente significantes $(p \leq 0,05)-$ Teste-t pareado

Legenda: $\mathrm{OD}$ = orelha direita; $\mathrm{OE}$ = orelha esquerda; Grupo $\mathrm{A}$ = grupo com fenilcetonúria e dieta adequada; Grupo I = grupo com fenilcetonúria e dieta inadequada; DP = desvio-padrão; Mín = valor mínimo observado; Máx = valor máximo observado

os déficits provocados pela PKU por meio de uma estimulação auditiva específica. Nesse contexto, a ação da Fonoaudiologia juntamente com a equipe de saúde envolvida (pediatras, nutricionistas e psicólogos), favorecerá um tratamento mais adequado, com maior benefício aos fenilcetonúricos.

\section{CONCLUSÃO}

As crianças com fenilcetonúria diagnosticadas e tratadas precocemente apresentaram piores limiares de audibilidade por via aérea, limiares de recepção de fala e índice de reconhecimento de fala evidenciados à audiometria tonal e vocal. Em relação à imitanciometria, as crianças com fenilcetonúria apresentaram limiares de reflexos estapedianos mais elevados quando comparadas às crianças normais.

Este estudo contribui para um melhor conhecimento sobre a audição de crianças com fenilcetonúria, incluindo aquelas que recebem tratamento precoce adequado. A equipe de saúde envolvida no tratamento dessas crianças (pediatras, nutricionistas, psicólogos e fonoaudiólogos) deve estar atenta às possíveis alterações, realizando os devidos encaminhamentos e melhorando a qualidade do tratamento que essas crianças recebem.

\section{AGRADECIMENTOS}

Agradecemos ao Núcleo de Ações e Pesquisa em Apoio Diagnóstico (NUPAD) da Faculdade de Medicina da Universidade Federal de Minas Gerais pelas bolsas concedidas às acadêmicas do Curso de Fonoaudiologia, e à Coordenação de Aperfeiçoamento de Pessoal de Nível Superior (CAPES) pelo apoio concedido para a realização dessa pesquisa, sob processo número 1023/07-1.

\begin{abstract}
Purpose: To investigate the existence of hearing impairments in infants with phenylketonuria with early diagnose and treatment, and to compare the audiological findings with those of their normal peers. Methods: Vocal and pure-tone audiometry and acoustic immitance tests were conducted in 63 children, 30 from a control group, with mean age of 8.1 years, and 33 from a study group, with phenylketonuria and mean age of 7.7 years. The study group was subdivided according to diet control: 15 subjects had adequate (mean age of 8.1 years) and 18 had inadequate diet control (mean age 7.2 years). Statistical analysis used t-test or ANOVA. Results: Audiometry showed that, in the control group, $83.3 \%$ of the subjects had normal hearing, and $16.7 \%$ had uni- or bilateral conductive hearing loss. In the study group, $66.7 \%$ of the subjects presented normal hearing, and $33.3 \%$ had conductive hearing loss. Immitance measures showed normal results in $91.7 \%$ of the children from the control group, and in $72.7 \%$ of the subjects with phenylketonuria. Differences were found between the groups regarding hearing thresholds, acoustic reflexes, speech reception thresholds, and speech recognition. No differences were observed between the results of phenylketonuria subjects with adequate and inadequate diets. Conclusion: Children with phenylketonuria early diagnosed and treated presented worse conductive hearing thresholds, speech reception threshold and speech recognition when compared to their normal peers, as evidenced in pure-tone and vocal audiometry.
\end{abstract}

Keywords: Hearing; Phenylketonurias; Hearing disorders; Acoustic impedance tests; Hearing loss; Audiometry 


\section{REFERÊNCIAS}

1. Malloy-Diniz LF, Cardoso-Martins C, Carneiro KC, Cerqueira MMM, Ferreira APA, Aguiar MJB, Starling AL. Funções executivas em crianças fenilcetonúricas: variações em relação ao nível de fenilalanina. Arq Neuropsiquiatr. 2004;62(2-B):473-9.

2. Starling ALP, Aguiar MJB. Fenilcetonúria. In: Leão E, Corrêa EJ, Mota JAC, Viana MB. Pediatria ambulatorial. 4a ed. Belo Horizonte: COOPMED; c2005. p. 69-70.

3. Scriver CR, Beaudet AL, Valle D, Sly WS, Childs B, Kinzler KW, Vogelstein B, editors. The metabolic and molecular bases of inherited disease. 8th ed. New York: McGraw-Hill; 2001. p. 1667-724.

4. Shaw V, Lawson M, editors. Clinical paediatric dietetics. London: Blackwell Science, Inc. 1994. p. 177-209.

5. Mira NVM, Marquez UML. Importância do diagnóstico e tratamento da fenilcetonúria. Rev Saúde Pública = J Public Health. 2000;34(1):86-96.

6. Kanufre VC, Santos JS, Soares RD, Starling ALP, Aguiar MJB. Abordagem dietética para fenilcetonúria. Rev Med Minas Gerais. 2001;11(3):129-34.

7. Recommendations on the dietary management of phenylketonuria. Report of Medical Research Council Working Party on Phenylketonuria. Arch Dis Child. 1993;68(3):426-7.

8. Starling ALP, Aguiar MJB, Kanufre VC, Soares SF. Fenilcetonúria. Rev Med Minas Gerais. 1999;9(3):106-10.

9. Bickel H, Bugard P, Link R. eds. Phenylketonuria, an international survey of management over 40 years. Proceedings of a workshop. Fulda, November 16-19, 1994. Eur J Pediatr. 1996:155 Suppl 1:S1-180.

10. Martins SRR. Incidência de fenilcetonúria e outras hiperfenilalaninemias no estado de Minas Gerais: dados do Programa Estadual de Triagem Neonatal [dissertação]. Belo Horizonte: Universidade Federal de Minas Gerais. Faculdade de Medicina; 2005.

11. Diamond A, Prevor MB, Callender G, Druin DP. Prefrontal cortex cognitive deficits in children treated early and continuously for PKU. Monogr Soc Res Child Dev. 1997;62(4):i-iv, 1-208.
12. Banich MT, Passarotti AM, White DA, Nortz MJ, Steiner RD. Interhemispheric interaction during childhood: II. Children with earlytreated phenylketonuria. Dev Neuropsychol. 2000;18(1): 53-71.

13. Anderson PJ, Wood SJ, Francis DE, Coleman L, Anderson V, Boneh A. Are neuropsychological impairments in children with early-treated phenylketonuria (PKU) related to white matter abnormalities or elevated phenylalanine levels? Dev Neuropsychol. 2007;32(2):645-68.

14. Centerwall SA, Centerwall WR. The discovery of phenylketonuria: the story of a young couple, two retarded children, and a scientist. Pediatrics. 2000;105(1 Pt 1):89-103.

15. Little SW. Suspected hearing defects in phenylketonuria. Arch Otolaryngol. 1962;75:515-8.

16. Gassió R, Fusté E, López-Sala A, Artuch R, Vilaseca MA, Campistol J. School performance in early and continuously treated phenylketonuria. Pediatr Neurol. 2005;33(4):267-71.

17. Jerger J. Clinical experience with impedance audiometry. Arch Otolaryngol. 1970;92(4):311-24.

18. Northern JL, Downs MP. Hearing in children. 4th ed. Baltimore: Williams \& Wilkins; c1991.

19. Lubianca Neto JF, Hemb L, Silva DB. Fatores de risco para otite média aguda recorrente: onde podemos intervir? - uma revisão sistemática da literatura. J Pediatr (Rio J). 2006;82(2):87-96.

20. Carvallo RMM. Medidas eletroacústicas da audição: A- imitância acústica. In: Limongi SCO. Fonoaudiologia. Informação para a formação: linguagem: desenvolvimento normal, alterações e distúrbios. Rio de Janeiro: Guanabara Koogan; 2003. p. 1-22.

21. Meneguello J, Domenico MLD, Costa MCM, Leonbardt FD, Barbosa LHF, Pereira LD. Ocorrência de reflexo acústico alterado em desordens do processamento auditivo. Rev Bras Otorrinolaringol. 2001;67(6):8305. 